\title{
Index Theorem and Equivariant Cohomology on the Loop Space *
}

\author{
Jean-Michel Bismut \\ Département de Mathématique, Université Paris-Sud, F-91405 Orsay, France
}

\begin{abstract}
In this paper, extending ideas of Witten and Atiyah, we describe some relations of equivariant cohomology on the loop space of a manifold to the path integral representation of the index of the Dirac operator on a twisted spin complex. In particular, the natural extension of the Chern character to the whole loop space is described. Also it is shown that the non-zero free homotopy classes of the loop space have 0 measure for the index measures associated to the index problem.
\end{abstract}

In [1], Atiyah, elaborating on an idea of Witten, suggested a very interesting interpretation of the path integral representation of the index of the Dirac operator on the spin complex of a spin manifold $M$. Namely, he wrote formally the index as the integral of a differential form on the loop space, which is equivariantly closed for the standard $S_{1}$ action on the loop space. By applying in this situation the prescriptions of equivariant cohomology (as expressed in a formula of Duistermaat-Heckman [11]) he obtains the index as the integral of a differential form over the fixed point set of the $S_{1}$ action, which is the manifold $M$, and so obtains the $\hat{A}$ genus of Hirzebruch.

The purpose of this paper is to describe a series of facts which confirm that the prescriptions of equivariant cohomology are indeed correct. Moreover, we extend Atiyah's formalism to the general Index theorem, i.e. we show that the path integral representation of the index of a Dirac operator on a twisted spin complex is given by an equivariantly closed form. In particular, we describe what is the natural extension of the Chern character of a fiber bundle over $M$ as an equivariantly closed form on the loop space, this extension being the one which is suggested by the path integral representation of the index.

In Sect. 1, we essentially prove (rigorously) that if $M$ has a finite homotopy group, the index measure of each non-trivial free homotopy class is exactly 0 . This extends our results in [7]. The proof uses the index theorem in an elementary way.

* This work was partially supported by the NSF grant MCS-8108814(A02) while the author was visiting the Institute for Advanced Study (Princeton) 
In Sect. 2, we obtain the formula for the index of a general Dirac operator as the integral of a differential form over the loop space. This section is entirely formal, but it uses heavily the trick which we had given in [7] to obtain the full index theorem for the Dirac operator, and which separates the $\hat{A}$ genus and the Chern character of the auxiliary bundle. It turns out that this procedure permits us to produce the adequate breaking of the (rigorous) path integral representation of the index into a (non-rigorous) representation as the integral of a differential form on the loop space, which is also in product form. Most of this section is conjectural. We are still far from any "proof" that the ideas of equivariant cohomology can be used rigorously in this framework.

In Sect. 3, we prove (rigorously) that the differential form which we produced in Sect. 2 is equivariantly closed on the loop space. It is obtained as an equivariant characteristic class of an infinite dimensional fiber bundle over the loop space.

The reader is referred to Atiyah's article [1] for an introduction to the subject, and also to our paper [7], from which the key ingredient of Sect. 2 is taken.

\section{The Index Measure and the Free Homotopy Classes in the Loop Space}

In this section, we give a few results which confirm that at least formally, the results predicted by the application of equivariant cohomology to the index measures on the loop space are in fact true. Namely, we essentially prove that if $M$ has a finite fundamental group, the index measure of each non-zero free homotopy class is exactly 0 .

We follow closely the presentation in our paper [7]. In a) the basic assumptions and notations are given. In b), the index measure is defined. In c) its elementary properties are described. In d) the results on the free homotopy classes are proved.

\section{a) Assumptions and Notations}

We will closely follow the notations in our paper [7]. $M$ denotes a $C^{\infty}$ compact connected oriented Riemannian manifold of even dimension $n=2 l$. TM denotes it tangent bundle, $T^{*} M$ the cotangent bundle. $T M$ and $T^{*} M$ are identified by the metric.

$N$ is the $\mathrm{SO}(n)$ principal bundle of oriented orthogonal frames in TM. For every $x \in M$, the fiber $N_{x}$ is the set of linear oriented isometries from $R^{n}$ (which is oriented by its canonical Euclidean base $e_{1}, \ldots, e_{n}$ ) into $T_{x} M . \pi$ is the projection $N \rightarrow M$.

We assume that $M$ is a spin manifold, i.e. the $\mathrm{SO}(n)$ principal bundle $N$ lifts to a $\operatorname{Spin}(n)$ principal bundle $N^{\prime}$ so that the projection $N^{\prime} \underset{\sigma}{\longrightarrow} N$ induces the covering mapping $\operatorname{Spin}(n) \underset{\sigma}{\longrightarrow} \mathrm{SO}(n)$ on each fiber.

Let $S$ be the $2^{l}$-dimensional Hermitian space of spinors, which splits into $S=S_{+} \oplus S_{-}$, where $S_{+}, S_{-}$are the spaces of positive and negative spinors. $S_{+}$and $S_{-}$have dimensions $2^{l-1}$ and are orthogonal in $S$. Moreover, $\operatorname{Spin}(n)$ acts irreducibly and unitarily on $S_{+}, S_{-}$.

Let $F, F_{+}, F_{-}$be the bundles of spinors over $M$

$$
F=N^{\prime} \times_{\operatorname{Spin}(n)} S, \quad F_{ \pm}=N^{\prime} \times_{\operatorname{Spin}(n)} S_{ \pm} .
$$


For every $x \in M$, the fiber $N_{x}^{\prime}$ can be identified to a set of unitary operators from $S$ into $F$, which send $S_{ \pm}$into $F_{ \pm}$.

Let $\theta$ be the $R^{n}$-valued one form defined by

$$
X \in T_{u}(N) \rightarrow \theta(X)=u^{-1} \pi_{*} X .
$$

Let $\mathscr{A}$ be the Lie algebra of $\mathrm{SO}(n)$, i.e. the set of $(n, n)$ real antisymmetric matrices. $\mathscr{A}$ is also the Lie algebra of $\operatorname{Spin}(n)$.

On $N$, we put the Levi-Civita connection, whose $\mathscr{A}$ valued connection form is denoted $\omega$. We know that

$$
d \omega=-\omega \wedge \omega+\Omega,
$$

where $\Omega$ is the equivariant representation of the curvature tensor $R$.

The Levi-Civita connection on $N$ lifts naturally to $N^{\prime}$. The corresponding connection form is $\sigma^{*} \omega$.

Let $\xi$ be a $k$-dimensional complex Hermitian bundle over $M . X$ denotes the $U(k)$ principal bundle of unitary frames in $\xi$. We assume that $X$ is endowed with a connection.

If $\mathscr{U}(k)$ is the Lie algebra of $U(k)$, let $\lambda$ be the $\mathscr{U}(k)$-valued connection form. We know that

$$
d \lambda=-\lambda \wedge \lambda+\Lambda,
$$

where $\Lambda$ is the equivariant representation of the curvature tensor $L$.

$\nabla$ denotes the covariant differentiation operator for any of the considered connections.

If $G$ is a vector bundle over $M, \Gamma(G)$ is the vector space of $C^{\infty}$ sections of $G$.

$D$ is the Dirac operator, which sends $\Gamma(F \otimes \xi)$ into itself and interchanges $\Gamma\left(F_{+} \otimes \xi\right)$ and $\Gamma\left(F_{-} \otimes \xi\right) . D_{ \pm}$is the restriction of $D$ to $\Gamma\left(F_{ \pm} \otimes \xi\right)$.

Definition 1.1. The index of $D_{+}$is defined by

$$
\text { Ind } D_{+}=\operatorname{dim} \operatorname{Ker} D_{+}-\operatorname{dim} \operatorname{Ker} D_{-} .
$$

For $t>0$, let $P_{t}(x, y)$ be the smooth kernel of $e^{-t D^{2} / 2}$. If $h \in \Gamma(F \otimes \xi)$, then

$$
c^{-t D^{2} / 2} h(x)=\int_{M} P_{t}(x, y) h(y) d y .
$$

In (1.4), $d y$ is the Riemannian volume in $M$. For $x, y \in M, P_{t}(x, y)$ sends $\Gamma\left(F_{ \pm} \otimes \xi\right)_{y}$ in $\Gamma\left(F_{ \pm} \otimes \xi\right)_{x}$.

If $A$ is a linear operator acting on $\left(F_{+} \otimes \xi\right)_{x}$ [respectively $\left.\left(F_{-} \otimes \xi\right)_{x}\right], \varrho_{+}(A)$ [respectively $\left.\varrho_{-}(A)\right]$ denotes its trace.

The following result is the base of the heat equation method.

Theorem 1.2. For any $t>0$

$$
\text { Ind } D_{+}=\int_{M}\left[\varrho_{+}\left(P_{t}(x, x)\right)-\varrho_{-}\left(P_{t}(x, x)\right)\right] d x .
$$

\section{b) The Index Measures}

We now describe shortly the index measures associated to the index problem.

$W$ is the set of continuous functions defined on $R^{+}$with values in $R^{n}$, whose standard element is $w_{t}=\left(w_{t}^{1}, \ldots, w_{t}^{n}\right) . P$ denotes the Wiener measure on $W$, with 
$P\left(w_{0}=0\right)=1 . d w$ will denote the differential of $w$ in the sense of Stratonovitch, $\delta w$ its differential in the sense of Itô (see [6]).

$X_{1}, \ldots, X_{n}$ are the standard horizontal vector fields on $N$ defined by

$$
\theta\left(X_{i}\right)=e_{i}, \quad \omega\left(X_{i}\right)=0 .
$$

$t$ is a positive real number.

Take $x_{0} \in M, u_{0} \in N_{x_{0}}$. Consider the stochastic differential equation

$$
d u_{s}=\sum_{1}^{n} X_{i}(u) \sqrt{t} d w^{i}, \quad u(0)=u_{0} .
$$

If $x_{s}=\pi u_{s}$, it is well known [7] that $x_{s / t}$ is exactly the Brownian motion on $M$, and $u_{s}$ is the parallel transport of $u_{0}$ along $x$.

$\tau_{s}^{0}$ denotes the parallel transport operator from vector fibers over $x_{0}$ to vector fibers over $x_{s}$, and $\tau_{0}^{s}$ is defined by $\tau_{0}^{s}=\left[\tau_{s}^{0}\right]^{-1}$.

Definition 1.3. $U_{s}$ is the process of linear mappings from $(F \otimes \xi)_{x_{0}}$ into itself defined by the equation

$$
\frac{d U}{d s}=-\frac{t}{2} U_{s}\left[\sum_{i<j}\left(u_{0} e_{i}\right)\left(u_{0} e_{j}\right) \otimes \tau_{0}^{s} L_{x_{s}}\left(u_{s} e_{i}, u_{s} e_{j}\right)\right] ; \quad U(0)=I .
$$

In (1.8), $e_{1}, \ldots, e_{n}$ is the canonical oriented base of $R^{n}$, and $\left(u_{0} e_{i}\right)\left(u_{0} e_{j}\right)$ acts on $F_{x_{0}}$ by Clifford multiplication.

Let $K$ be the scalar curvature of $M$. We now have the result of [7, Theorem 2.5].

Theorem 1.4. For any $h \in \Gamma(F \otimes \xi)$,

$$
e^{-t D^{2} / 2} h\left(x_{0}\right)=E^{P}\left[\exp \left\{-\int_{0}^{1} \frac{t K\left(x_{s}\right)}{8} d s\right\} U_{1} \tau_{0}^{1} h\left(x_{1}\right)\right] .
$$

Definition 1.5. For $y_{0} \in M, Q_{x_{0}, y_{0}}^{t}$ denotes the law of $x$ conditional on $x_{1}=y_{0}$.

For the precise definition of $Q_{x_{0}, y_{0}}^{t}$, see [8, Sect. 2].

Let $p_{t}(x, y)$ denote the $C^{\infty}$ kernel of $e^{t \Delta / 2}$ (where $\Delta$ is the Laplace-Beltrami operator on $M$ ).

From Theorem 1.4, we immediately get:

Theorem 1.6. For any $x_{0}, y_{0} \in M$,

$$
P_{t}\left(x_{0}, y_{0}\right)=E^{Q_{x_{0}, y_{0}}^{t_{0}}}\left[\exp \left\{-t \int_{0}^{1} \frac{K\left(x_{s}\right)}{8} d s\right\} U_{1} \tau_{0}^{1}\right] p_{t}\left(x_{0}, y_{0}\right) .
$$

Proof. A.e. equality in (1.10) is obvious by disintegrating (1.9). The left-hand side is $C^{\infty}$ in $y_{0}$. Moreover, the results of $[8$, Sect. 2] (see in particular the proof of Theorem 2.14 in [8]) show that the right-hand side of (1.10) is continuous in $y_{0}$. Equation (1.10) then holds for every $y_{0}$.

We are now ready to define the index measure.

Definition 1.7. $\bar{M}^{0}$ denotes the set of continuous mappings $x$. defined on $S_{1}=R / Z$ with values in $M$. For $s \in S_{1}, k_{s}$ denotes the mapping

$$
x \in \bar{M}^{0} \rightarrow k_{s} x .=x_{s+} .
$$


Note that for any $x_{0}, Q_{x_{0}, x_{0}}^{t}$ is a probability measure on $\bar{M}^{0}$.

We finally define the index measure.

Definition 1.8. $R^{t}$ denotes the bounded signed measure on $\bar{M}^{0}$ which is such that if $h$ is a bounded Borel function on $\bar{M}^{0}$, then

$$
\begin{array}{rl}
\int_{\bar{M}^{0}} & h(x .) d R^{t}(x) \\
= & \int_{M} E^{\left.Q t_{x_{0}}, x_{0}\right)}\left[\exp \left\{-t \int_{0}^{1} \frac{K\left(x_{s}\right) d s}{8}\right\}\left[\varrho_{+}\left(U_{1} \tau_{0}^{1}\right)-\varrho_{-}\left(U_{1} \tau_{0}^{1}\right)\right] h\left(x_{.}\right)\right] \\
& \cdot p_{t}\left(x_{0}, x_{0}\right) d x_{0} .
\end{array}
$$

c) Some Elementary Properties of the Index Measures

We start giving some trivial properties of the measures $R^{t}$.

Theorem 1.9. For any $t>0$,

$$
\int_{\bar{M}^{0}} d R^{t}(x)=\operatorname{Ind} D_{+} .
$$

For any $s \in R / Z, R^{t}$ is invariant under $k_{s}$.

Proof. (1.13) is an obvious consequence of Theorems 1.2 and 1.6.

We claim that the measure $S^{t}$ on $\bar{M}^{0}$ defined by

$$
\int_{M^{0}} h(x) d S^{t}(x)=\int_{M} E^{Q_{x_{0}}, x_{0}} h(x) p_{t}\left(x_{0}, x_{0}\right) d x_{0}
$$

is invariant under $k_{s}$, i.e. that

$$
\int_{\bar{M}^{0}} h\left(k_{s} x\right) d S^{t}(x)=\int h(x) d S^{t}(x) .
$$

We only need to prove (1.15) when $h$ is given by

$$
h(x)=f_{1}\left(x_{t_{1}}\right) \ldots f_{m}\left(x_{t_{m}}\right) ; \quad 0 \leqq t_{1}<t_{2} \ldots<t_{m} \leqq 1 .
$$

Now if $h$ is given by (1.16), we see easily that the left-hand side of (1.16) is given by

$$
\int_{M^{m}} f_{1}\left(x_{1}\right) \ldots f_{m}\left(x_{m}\right) p_{t_{2}-t_{1}}\left(x_{1}, x_{2}\right) \ldots p_{t_{1}-t_{m}}\left(x_{m}, x_{1}\right) d x_{1} \ldots d x_{m},
$$

where $t_{1}-t_{m}$ is calculated in $R / Z$. Equation (1.15) is now obvious.

$\int_{0}^{1} K\left(x_{u}\right) d u$ is clearly invariant under $k_{s}$.

Finally, using self-explanatory notations, it is trivial to prove that

$$
\left(U_{1} \tau_{0}^{1}\right) \circ k_{s}=\tau_{s}^{0} U_{s}^{-1} U_{1} \tau_{0}^{1} U_{s} \tau_{0}^{s}
$$

[where $\left(U_{1} \tau_{0}^{1}\right) \circ k_{s}$ sends $(F \otimes \xi)_{x_{s}}$ into itself] so that

$$
\left[\varrho_{+}\left(U_{1} \tau_{0}^{1}\right)-\varrho_{-}\left(U^{1} \tau_{0}^{1}\right)\right] \circ k_{s}=\varrho_{+}\left(U_{1} \tau_{0}^{1}\right)-\varrho_{-}\left(U_{1} \tau_{0}^{1}\right) .
$$

The proof is finished. 


\section{d) The Index Measure of Free Homotopy Classes}

Take $x_{0} \in M$. Let $\pi_{1}(M)$ denote the homotopy group with base point $x_{0} \cdot e$ denotes the unit element in $\pi_{1}(M)$.

The connected components of $\bar{M}^{0}$ are exactly the free homotopy classes in $\bar{M}^{0}$. Moreover, it is well known that to each free homotopy class, we can associate one and only one conjugacy class in $\pi_{1}(M)$. Finally, each free homotopy class is stable under $k_{s}$.

Let $\Lambda_{0}$ be the set of loops in $\bar{M}^{0}$ which are (freely) homotopic to 0 .

We now have the following extensions of [7, Theorem 3.21]:

Theorem 1.10. Assume that $\pi_{1}(M)$ is finite. Then for any free homotopy class $\Lambda \neq \Lambda_{0}$,

$$
R_{t}(\Lambda)=0 .
$$

Proof. We give two simple proofs.

Proof No. 1. Let $\tilde{M}$ be the universal covering of $M . \tilde{M}$ is a $\pi_{1}(M)$-bundle over $M$. Let $(T, V)$ be a finite dimensional unitary representation of $\pi_{1}(M)$ so that if $a \in \pi_{1}(M), T(a)$ acts linearly on $V$. Let $V^{\prime}$ denote the corresponding flat bundle over $M$. We may define the Dirac operator $D^{V^{\prime}}$ on $F \otimes \xi \otimes V^{\prime}$. From the index theorem we know that

$$
\operatorname{Ind} D_{+}^{V^{\prime}}=(\operatorname{dim} V) \operatorname{Ind} D_{+} .
$$

If $\chi_{V}(a)$ denote the trace of $T(a)$, if $\tau_{s}^{\prime 0}$ is the parallel transport operator on the fiber of $V^{\prime}$ along $x$, since $V^{\prime}$ is flat, Theorem 1.9 shows that

$$
\text { Ind } D_{+}^{V^{\prime}}=\int \chi_{V}\left(\tau_{0}^{\prime 1}\right) d R_{t} \text {. }
$$

Since $\operatorname{dim} V=\chi_{V}(e)$, from (1.21), (1.22), we see that

$$
\int \chi_{V}\left(\tau_{0}^{\prime 1}\right) d R_{t}=\chi_{V}(e) \operatorname{Ind} D_{+} .
$$

For $x \in \bar{M}^{0}$, let $h(x)$ be the conjugacy class in $\pi_{1}(M)$ associated to $x$. Since the $\chi_{V}$ span linearly the central functions on $\pi_{1}(M)$, we see from (1.23) that for any central function $g$ on $\pi_{1}$,

$$
\int g(h(x)) d R_{t}=g(e) \operatorname{Ind} D_{+} .
$$

By taking $g$ to be the characteristic function of the conjugacy class corresponding to $A$, we obtain (1.20).

Proof No. 2. $\pi_{1}(M)$ acts freely on $\tilde{M}$, and so to each $a \in \pi_{1}(M)$, we may associate the mapping $\tilde{x} \in \tilde{M} \rightarrow \tilde{x} a \in \tilde{M}$.

We may lift $F$ and $\xi$ to $\tilde{M}$, and define the associated Dirac operator $\tilde{D}$ on the lifted bundle $\tilde{F} \otimes \tilde{\xi}$.

Now every $a \in \pi_{1}(M)$ acts isometrically on $\tilde{M}$, preserves the spin structure of $\tilde{M}$ and also acts unitarily on $\tilde{\xi}$. We can then define the Lefschetz number $L(a)$.

Take $a \in \pi_{1}(M) \neq e$. Since $a$ does not have fixed points in $\tilde{M}$, it follows from the Lefschetz fixed point theorem in Atiyah-Bott [2] that $L(a)=0$.

For $\Lambda \neq \Lambda_{0}$, let $l$ denote the corresponding conjugacy class in $\pi_{1}(M)$. Since $l$ does not contain $e$, it is clear that

$$
\sum_{a \in l} L(a)=0 .
$$


Now using the probabilistic representation of the Lefschetz number in [7, Sect. 4], it is not difficult to prove that if $h$ is the number of elements in $\pi_{1}(M)$, then

$$
\sum_{a \in l} L(a)=h \int 1_{x \in \Lambda} d R_{t}(x),
$$

the factor $h$ coming from the integration over $\tilde{M}$ which is an $h$-fold covering of $M$.

From (1.25), (1.26), the theorem follows.

Let now $M^{\prime}$ be any covering of $M$. For each $x \in \bar{M}^{0}$, we may define the monodromy of $x$ which maps the fiber $M_{x_{0}}^{\prime}$ into itself.

Corollary. Assume that $\pi_{1}(M)$ is finite. Let $\Lambda^{\prime}$ be the set of $x \in \bar{M}^{0}$ whose monodromy is not the identity mapping. Then

$$
R_{t}\left(\Lambda^{\prime}\right)=0 \text {. }
$$

Proof. It is easy to see that $\Lambda^{\prime}$ is a finite union of non-zero free homotopy classes. The corollary is now obvious by Theorem 1.10.

Remark 1. It should be pointed out that the proofs of Theorem 1.10 involve arguments which are easily obtainable by heat equation methods, i.e. do not necessitate the explicit cohomological formulas of Atiyah and Bott [2], Atiyah and Singer [3]. Essentially, once it is known - say by spectral theory - that a quantity an index or a Lefschetz number - is given by the integral of a function depending on $t>0$, the fact that this number is 0 is in general easy to show by studying the asymptotics as $t \downarrow \downarrow 0$ of the corresponding integral.

Remark 2. The results of Theorem 1.10 can be easily extended to the case, where, instead of considering the universal covering $\tilde{M}$, we consider any Galois covering, which should then be compact.

When $\pi_{1}(M)$ is not finite, we can prove the results of the first part of Theorem 1.10 hold only if there is a decreasing sequence of normal subgroups $H_{n}$ of $\pi_{1}(M)$ such that $\cap H_{n}=\{e\}$ and that for any $n \in N, \pi_{1}(M) / H_{n}$ is finite.

However, the following result is true in full generality.

Theorem 1.11. The following formula holds:

$$
\text { Ind } D_{+}=\int_{\bar{M}^{0}} 1_{x \in \Lambda_{0}} d R_{t}
$$

Proof. $\tilde{M}$ still denotes the universal covering of $M . \pi$ is the covering projection $\tilde{M} \rightarrow M$. Let $\tilde{D}$ be the lift of $D$ to $M$, and let $\widetilde{P}_{t}$ be the smooth kernel of $\widetilde{P}_{t}$ over $\tilde{M}$. Let $F$ be a fundamental domain in $\tilde{M}$. Now it essentially follows from the AtiyahSinger $L_{2}$ index theorem [5] that

$$
\operatorname{Ind} D_{+}=\int_{F}\left[\varrho_{+}\left(\widetilde{P}_{t}(x, x)\right)-\varrho_{-}\left(\tilde{P}_{t}(x, x)\right)\right] d x .
$$

By proceeding as in [7, Theorem 3.21], we see that the right-hand side of (1.29) is exactly

$$
\int_{\bar{M}^{0}} 1_{x \in \Lambda_{0}} d R_{t}(x) .
$$

(1.28) is then proved. 


\section{Equivariant Cohomology and the Index Theorem}

In [1], Atiyah, following Witten, has shown how it was possible to write formally the path integral which gives the index of the Dirac operator on the spin complex of a spin manifold as the integral of a formal differential form. It turns out that this form is equivariantly closed for the standard $S_{1}$ action on the loop space, and that the application of equivariant cohomology formulas (adequately renormalized) produces the right cohomological expression for the index, i.e. the $\hat{A}$ polynomial of Hirzebruch.

In this section, we show how to write formally the index of the Dirac operator $D$ as the integral of a differential form, which as we shall see in Sect. 3, is also equivariantly closed.

In fact, let us recall that in [7], for the proof of the index theorem, we had introduced an auxiliary Brownian motion with values in $\mathscr{A}$, whose effect was to break the probabilistic expression of the index, and obtain the factorization of the cohomological formula for the index as the product of the $\hat{A}$ genus and of the Chern character. It turns out that the same trick produces an expression for the index as the formal integral of a differential form on the loop space which is the product of the Witten-Atiyah form [1] and of another form which extends the Chern character to the loop space (although it is not a Chern character).

Everything which follows is essentially formal. Expressions which might look horrendous to probabilists are written, as well as diverging infinite products. However, as we shall see in Sect. 3, what we produce is algebraically quite sound, and predicts the right answers for the index theorem.

In a) notations and assumptions are given. In b) the observations of Witten and Atiyah are briefly summarized. In c) a conjectural formula is given for the index. In the whole section, we closely follow our computations in [7]. The reader who is not familiar with the stochastic calculus can consult [6].

\section{a) Assumptions and Notations}

The notations are very much the same as in Sect. 1 . However, we will work with smooth loops instead of working with continuous loops. The reason is that standard operations like parallel transport are well-defined along any smooth loop, while they are only defined in a measure theoretic sense along Brownian loops. Of course, we will have always to keep in mind that the measures we will talk about give 0 measure to smooth loops.

Definition 2.1. $\bar{M}^{\infty}$ denotes the set of $C^{\infty}$ mappings $s \in S_{1}=R / Z \rightarrow x_{s} \in M$.

If $x \in \bar{M}^{\infty}$, the tangent space $T_{x} \bar{M}^{\infty}$ is identified to the space of smooth periodic vector fields $X$ over $x$ so that $X_{s} \in T_{x_{s}} M$.

If $X, Y \in T_{x} \bar{M}^{\infty}$, we define the scalar product

$$
\langle X, Y\rangle=\int_{0}^{1}\left\langle X_{s}, Y_{s}\right\rangle d s .
$$

$\bar{M}^{\infty}$ is then a prehilbertian manifold.

Now $S_{1}$ acts isometrically on $\bar{M}^{\infty}$ by the mappings $k_{s}$ defined by

$$
k_{s} x=x_{s+\cdot}
$$


$\left\{k_{s}\right\}_{s \in S_{1}}$ is a group of isometries, whose generating Killing vector field $X$ is given by

$$
(X(x))_{s}=\left(\frac{d x}{d s}\right)_{s}
$$

$E$ denotes the exterior algebra of $T^{*} \bar{M}^{\infty}$. Of course, $E$ decomposes into

$$
E=\bigoplus_{0}^{+\infty} E^{p}
$$

where $E^{p}$ is the set of $p$-differential forms over $\bar{M}^{\infty}$.

If $x \in \bar{M}^{\infty}$, one $\mu \in E_{x}$ is defined by a formal series

where $\mu_{p} \in E_{x}^{p}$.

$$
\mu=\sum_{0}^{+\infty} \mu_{p}
$$

$\Gamma(E)$ denotes the set of $C^{\infty}$ sections of $E . d$ denotes exterior differentiation, $i_{X}$ the usual interior product by $X$. Recall that

$$
L_{X}=\left(d+i_{X}\right)^{2} .
$$

Definition 2.2. $\mu \in \Gamma(\bar{\Lambda})$ will be said to be equivariantly closed if

$$
\left(d+i_{X}\right) \mu=0 .
$$

Following Witten, Atiyah [1], we define the fundamental one-form $\alpha$.

Definition 2.3. $\alpha$ is the one form dual to $X$, i.e. if $x \in \bar{M}^{\infty}, Y \in T_{x} \bar{M}^{\infty}$

$$
\alpha(Y)=\langle X, Y\rangle .
$$

Since $X$ is a Killing vector field, it is clear that

$$
L_{X} \alpha=0 \text {. }
$$

Using (2.4), (2.7) it is obvious that $\left(d+i_{X}\right) \alpha$ is equivariantly closed.

We now have [1].

Theorem 2.4. $\frac{d \alpha}{2}$ is the two form $\sigma$ defined for $Y, Z \in T_{x}\left(\bar{M}^{\infty}\right)$ by

$$
\sigma(Y, Z)=\int_{0}^{1}\left\langle\frac{D Y}{D t}, Z\right\rangle d t
$$

$\frac{i_{x} \alpha}{2}$ is the energy functional $E$ defined by

$$
E(x)=\int_{0}^{1} \frac{\left|\dot{x}_{s}\right|^{2}}{2} d s .
$$

$E+\sigma$ is equivariantly closed.

b) The Observation of Witten and Atiyah on the Spin Complex

For greater clarity, we briefly recall the remark of Witten and Atiyah [1] on the path integral representation of the index of the Dirac operator on the spin complex. In this section, $\xi$ will then be the trivial line bundle. 
Take $x \in \bar{M}^{\infty}$. Consider the eigenvalue problem on $Y \in T_{x} \bar{M}^{\infty}$,

$$
\frac{D Y}{D t}=\lambda Y \text {. }
$$

Recall that $Y$ has to be periodic, i.e. $Y_{0}=Y_{1}$. If $\tau_{1}^{0}$ is the parallel transport operator from $T_{x_{0}} M$ into $T_{x_{1}} M$, we denote by $e^{ \pm i \theta_{j}}\left(1 \leqq j \leqq l ; 0 \leqq \theta_{j} \leqq \pi\right)$ its eigenvalues. It is then trivial to check that in (2.10), $\lambda$ can take the values

$$
\pm 2 i \pi m \pm i \theta_{j} \text {. }
$$

The Pfaffian of $-\sigma$ can then be written formally as

$$
P f(-\sigma)=\prod_{j=1}^{l}\left[\theta_{j} \prod_{m=1}^{+\infty}\left[4 \pi^{2} m^{2}-\theta_{j}^{2}\right]\right] .
$$

Now the infinite product (2.12) can be renormalized by dividing formally by $\prod_{m=1}^{+\infty}\left(4 \pi^{2} m^{2}\right)^{l}$, so that

$$
\frac{P f(-\sigma)}{\left(\prod_{1}^{+\infty} 4 \pi^{2} m^{2}\right)^{l}}=\prod_{1}^{l} 2 \sin \frac{\theta_{j}}{2} .
$$

Now it turns out that by $[2,3]$ if we still denote by $\tau_{1}^{0}$ the parallel transport operator from $\left(F_{ \pm}\right)_{x_{0}}$, into itself along $x$, and by $\tau_{0}^{1}$ its inverse, if $\chi_{ \pm}\left(\tau_{0}^{1}\right)$ is the corresponding trace, then

and so

$$
\chi_{+}\left(\tau_{0}^{1}\right)-\chi_{-}\left(\tau_{0}^{1}\right)= \pm(i)^{l} \prod_{1}^{l}\left(2 \sin \frac{\theta_{j}}{2}\right),
$$

$$
i^{l} \frac{P f(-\sigma)}{\left(\prod_{1}^{+\infty} 4 \pi^{2} m^{2}\right)^{l}}= \pm\left[\chi_{+}\left(\tau_{0}^{1}\right)-\chi_{-}\left(\tau_{0}^{1}\right)\right] .
$$

What follows is entirely formal. However, we will keep track of renormalization constants (which are possibly infinite). Moreover, we denote by $d$ the dimension of $\bar{M}^{\infty}(d=+\infty$ !).

The idea of Witten and Atiyah [1] is to interpret the measure $S^{t}$ defined in (1.14) in the form

$$
d S^{t}(x)=\frac{1}{(\sqrt{2 \pi t})^{d}} \exp \left\{-\int_{0}^{1} \frac{|\dot{x}|^{2}}{2 t} d s\right\} d D(x),
$$

where $d D(x)$ is a generalized Riemannian volume element in $\bar{M}^{\infty}$, so that by neglecting $\exp \left\{-\frac{t}{8} \int_{0}^{1} K\left(x_{s}\right) d s\right\}$ - which could be the result of a normalization procedure, and keeping track of the various normalization constants, formula (1.13) would be written

$$
\operatorname{Ind} D_{+}=\left(\frac{\left(\prod_{1}^{+\infty} m^{2}\right)^{l}}{(2 \pi)^{l}}\right) i^{l} \int \exp \left\{-\frac{E(x)}{t}\right\} \frac{P f(-\sigma)}{t^{d}} d D(x) .
$$


Now if $d$ was $<+\infty$, and $\mu=\left(\mu_{1} \ldots \mu_{d}\right) \in \Gamma(E)$, by definition $\int \mu$ is set to be equal to $\int \mu_{d}$.

By using the fact that if $d$ was $<+\infty$, then

$$
P f(\sigma) d D(x)=\frac{\sigma^{d}}{d !},
$$

and using the previous conventions in infinite dimensions, then

$$
\operatorname{Ind} D_{+}=\frac{\left(\prod_{1}^{+\infty} m^{2}\right)^{l} i^{l}}{(2 \pi)^{l}} \int \exp \left(-\frac{E(x)+\sigma}{t}\right) \text {. }
$$

$M$ is exactly the set of fixed points of the $k_{s}\left(s \in S_{1}\right)$.

Now Witten and Atiyah [1] noted that if the principles of equivariant cohomology extend in an infinite dimensional context, the right-hand side of (2.19) can be expressed as the integral of a differential form over $M$ (see Duistermaat and Heckman [11], Berline and Vergne [10], and Atiyah and Bott [4] for the finite dimensional case).

Explicit computations in [1] show that this differential form - which turns out to be the inverse of the equivariant Euler class of the normal bundle of $M$ in $\bar{M}^{\infty}$-is exactly the $\hat{A}$ genus of Hirzebruch, i.e. the right answer for $\operatorname{Ind} D_{+}$[3]. In particular, the fact that the right-hand side of (2.19) does not depend on $t$ is now a trivial consequence of the fact that $E$ and $\sigma$ are both 0 on $M$.

Although this reasoning is at the present stage entirely formal, it raises the exciting prospect that not only measures but also forms are relevant in infinite dimensional integration.

Moreover, the results of Theorem 1.10 - when applied to the spin complex - are perfectly compatible with the equivariant cohomology predictions, since $k_{s}$ acts on each $\Lambda \neq \Lambda_{0}$ without fixed points, and so equivariant cohomology predicts the integral should be 0 .

Now, as we shall see, there is an equivariant interpretation for the index of the Dirac operator on any twisted spin complex.

Remark 1. The equivariant cohomology of a space $F$ on which $S_{1}$ acts is the cohomology of the fibre space over the classifying space of $S_{1}$ with fiber $F$. In the case where $F$ is a finite dimensional compact manifold on which a vector field $Y$ induces an action of $S_{1}$, Witten [14] introduced the complex of $Y$ invariant forms with the differential $d+i_{Y}$. As shown in Atiyah and Bott [4], the construction of Witten produces a de Rham version of the equivariant cohomology of $F$.

In our case $F$ is the infinite dimensional manifold $\bar{M}^{\infty}$. Still the Witten complex is well-defined. However, the relation of the abstract equivariant cohomology of $\bar{M}^{\infty}$ to the Witten complex is no longer clear. Incidently, note that since we are talking about differential forms which can be integrated, the abstract cohomological model which is to be considered should be an extension of the compactly supported cohomology for finite dimensional manifolds. 
c) Identification of the Integrand for a Twisted Spin Complex

We now use the notations of Sect 1, as well as our results of [7]. Let us first say that what we do here is essentially trivial if the complex dimension of $\xi$ is 1 , so that what follows is relevant only if $\operatorname{dim} \xi \geqq 2$. Also we authorize ourselves several formal manipulations.

We first proceed (rigorously) as in [7].

Definition 2.5. $W^{\prime}$ denotes the set of continuous functions defined on $R^{+}$with values in $\mathscr{A}$. $P^{\prime}$ denotes the Wiener measure on $\mathscr{A}$, with $P^{\prime}(\gamma(0)=0)=1$.

Under $P^{\prime}$, the various $\left(\gamma_{j, s}^{i}\right)(i<j)$ are independent standard Brownian motions. $x_{s}, u_{s}, U_{s}$ are taken as in Sect. $\left.1 b\right)$.

We will now work on $\left(W \times W^{\prime}, P \otimes P^{\prime}\right)$, i.e. $(x, u, U)$ and $\gamma$ are independent.

Definition 2.6. $V_{s}^{1}$ is the process of linear mappings of $F_{x_{0}}$ into itself defined by the Itô equation

$$
\begin{aligned}
d V_{s}^{1} & =-\frac{t}{2} V_{s}^{1} \sum_{i<j}\left(u_{0} e_{i}\right)\left(u_{0} e_{j}\right) \delta \gamma_{i, s}^{j}, \\
V_{0}^{1} & =I .
\end{aligned}
$$

$V_{s}^{2}$ is the process of linear mappings from $\xi_{x_{0}}$ into itself defined by the Itô equation,

$$
d V_{s}^{2}=V_{s}^{2}\left(\sum_{i<j} \tau_{0}^{s} L_{x_{s}}\left(u_{s} e_{i}, u_{s} e_{j}\right) \delta \gamma_{i, s}^{j}\right), \quad V_{0}^{2}=I
$$

In what follows, $E^{P^{\prime}}$ denotes the expectation operator in the variable $\gamma$. for fixed $(x, u)$.

Proposition 2.7. The following relation holds:

$$
U_{1}=E^{P^{\prime}}\left[V_{1}^{1} \otimes V_{1}^{2}\right] \text {. }
$$

Proof. This is Proposition 3.7 in [7].

Clearly under the assumptions of Definition 1.8, which imply that $x_{s}$ is a loop, we have

$$
\varrho_{+}\left(U_{1} \tau_{0}^{1}\right)-\varrho_{-}\left(U_{1} \tau_{0}^{1}\right)=E^{P^{\prime}}\left[\left[\chi_{+}\left(V_{1}^{1} \tau_{0}^{1}\right)-\chi_{-}\left(V_{1}^{1} \tau_{0}^{1}\right)\right] \operatorname{Tr} V_{1}^{2} \tau_{0}^{1}\right]
$$

We will now try to interpret the factorization of the right-hand side of (2.23) in terms of exterior products of differential forms.

To simplify the discussion, we first assume that $\bar{\gamma}_{s}$ is a $C^{\infty}$ function defined on $[0,1]$ with values in $\mathscr{A}$ such that $\bar{\gamma}_{0}=0$, and that $\bar{V}_{s}^{1}, \bar{V}_{s}^{2}$ are the solutions of the differential equations,

$$
\begin{array}{ll}
d \bar{V}_{s}^{1}=-\frac{t}{2} \bar{V}_{s}^{1} \sum_{i<j}\left(u_{0} e_{i}\right)\left(u_{0} e_{j}\right) d \bar{\gamma}_{i, s}^{j}, & \bar{V}_{0}^{1}=I \\
d \bar{V}_{s}^{2}=\bar{V}_{s}^{2}\left(\sum_{i<j} \tau_{0}^{s} L_{x_{s}}\left(u_{s} e_{i}, u_{s} e_{j}\right) d \bar{\gamma}_{i, s}^{j}\right), & \bar{V}_{0}^{2}=I
\end{array}
$$

Consider the 2-form on $T_{x} \bar{M}^{\infty}$

$$
r^{\bar{\gamma}}(Y, Z)=\int_{0}^{1}\left\langle Y_{s}, u_{s} \dot{\bar{\gamma}}_{s} u_{s}^{-1} Z_{s}\right\rangle d s
$$


By recalling that $\frac{1}{2} \sum_{i<j} e_{i} e_{j} \dot{\gamma}_{i, s}$ is exactly the action of $\dot{\bar{\gamma}}_{s}$ on $S$ (see e.g. [7, Proposition 1.3]) we see easily that by using the same renormalization prescriptions as in (2.15), we have

$$
\frac{i^{l} P f\left[-\left(\sigma+\operatorname{tr}^{\bar{\gamma}}\right)\right]}{\left(\prod_{1}^{+\infty} 4 \pi^{2} m^{2}\right)^{l}}= \pm\left[\chi_{+}\left(\bar{V}_{1} \tau_{0}^{1}\right)-\chi_{-}\left(\bar{V}_{1} \tau_{0}^{1}\right)\right] .
$$

Now consider the equation in the sense of Stratonovitch

$$
d V_{s}^{\prime 1}=-\frac{t}{2} V_{s}^{\prime 1} \sum_{i<j}\left(u_{0} e_{i}\right)\left(u_{0} e_{j}\right) d \gamma_{i, s}^{j}, \quad V_{0}^{\prime 1}=I
$$

By $[7$, Theorem 3.10$]$ we know that

$$
V_{s}^{1}=\exp \frac{n(n-1) t^{2}}{16} V_{s}^{\prime 1}
$$

Moreover, since we know (see e.g. [9, Chap. I]) that Stratonovitch equations can be adequately approximated by standard differential equations, we will use (2.26) formally with $\gamma$ instead of $\bar{\gamma}, V^{\prime 1}$ instead of $\bar{V}^{1}$, do some stochastic calculus and eliminate $\gamma$.

Using Theorem 1.9, and the same normalization procedures as in (2.26), we find that

$$
\begin{aligned}
\operatorname{Ind} D_{+}= & \frac{\prod_{1}^{+\infty}\left(m^{2}\right)^{l}}{(2 \pi)^{l}} i^{l} \int \exp \left(-\frac{E(x)}{t}\right) \frac{P f\left[-\left(\sigma+\operatorname{tr}^{\gamma}\right)\right]}{t^{d}} \\
& \cdot \exp \frac{n(n-1) t^{2}}{16} \operatorname{Tr}\left[V_{1}^{2} \tau_{0}^{1}\right] d D(x) d P^{\prime}(\gamma) .
\end{aligned}
$$

Now observe that formally

$$
\frac{P f\left[-\left(\sigma+\operatorname{tr}^{\gamma}\right)\right]}{t^{d}}=P f\left[-\left(\frac{\sigma}{t}+r^{\nu}\right)\right] .
$$

Using the same conventions as in (2.19), we can then write

$$
\begin{aligned}
& \int \exp \left(-\frac{E(x)}{t}\right) \frac{P f\left[-\left(\sigma+\operatorname{tr}^{\gamma}\right)\right]}{t^{d}} \operatorname{Tr}\left[V_{1}^{2} \tau_{0}^{1}\right] d D(x) d P^{\prime}(\gamma) \\
& \quad=\int \exp -\left(\frac{E(x)+\sigma}{t}+r^{\gamma}\right) \operatorname{Tr}\left[V_{1}^{2} \tau_{0}^{1}\right] d P^{\prime}(\gamma) .
\end{aligned}
$$

Now since even forms form a commutative algebra, we have

$$
\exp -\left(\frac{E(x)+\sigma}{t}+r^{\gamma}\right)=\exp \left(-\frac{E(x)+\sigma}{t}\right) \exp \left(-r^{\gamma}\right) .
$$

We should now compute

$$
\int \exp \left\{-r^{\gamma}\right\} \operatorname{Tr} V_{1}^{2} \tau_{0}^{1} d P^{\prime}(\gamma)
$$


The computation is very close to what we did in [7, Theorem 3.18]. We will write formally

$$
r^{\gamma}=\int_{0}^{1} \sum_{i<j}\left[u_{s} d x_{s}^{i} \wedge u_{s} d x_{s}^{j}\right] \delta \gamma_{j, s}^{i} .
$$

In (2.34), $u_{s} d x_{s}^{i}$ is the one form at $T_{x_{s}} M$ such that if $Y \in T_{x_{s}} M$,

$$
\left\langle u_{s} d x_{s}^{i}, Y\right\rangle=\left[u_{s}^{-1} Y\right]^{i} \text {. }
$$

Set for $0 \leqq s \leqq 1$,

$$
r_{s}^{\gamma}=\int_{0}^{s} \sum_{i<j}\left(u_{v} d x_{v}^{i} \wedge u_{v} d x_{v}^{j}\right) \delta \gamma_{j, v}^{i} .
$$

The same argument as in [7, Theorem 3.18] shows that

$$
\exp \left(-r_{s}^{\gamma}\right)=1-\int_{0}^{s}\left(\exp -r_{v}^{\gamma}\right) \wedge \delta r_{v}^{\gamma}
$$

In fact, recall that $\exp \left(-r_{s}^{\gamma}\right)$ is calculated in an infinite dimensional exterior algebra, and that $\left[d x_{s}^{i} \wedge d x_{s}^{j}\right]^{2}=0$, so that the higher order terms in Itô's formula drop out.

Now by Itô's formula, we have

$$
\begin{aligned}
\exp \left(-r_{s}^{\gamma}\right) \otimes V_{s}^{2}= & 1 \otimes I+\int_{0}^{s}\left(\exp -r_{v}^{\gamma} \otimes V_{v}^{2}\right) \\
& \cdot\left[-\delta r_{v}^{\gamma} \otimes 1+1 \otimes \sum_{i<j} \tau_{0}^{v} L_{x_{v}}\left(u_{v} e_{i}, u_{v} e_{j}\right) \delta \gamma_{i, v}^{j}\right] \\
& +\int_{0}^{s} \exp \left(-r_{v}^{\gamma}\right) \otimes V_{v}^{2}\left[\left(u_{v} d x_{v}^{i} \wedge u_{v} d x_{v}^{j}\right) \otimes \tau_{0}^{v} L_{x_{v}}\left(u_{v} e_{i}, u_{v} e_{j}\right)\right] d v
\end{aligned}
$$

By taking expectations with respect to $\gamma$, we find

$$
\begin{aligned}
E^{P^{\prime}}\left[\exp -r_{s}^{\gamma} \otimes V_{s}^{2}\right]= & 1 \otimes I+\int_{0}^{s} E^{P^{\prime}}\left[\left(\exp -r_{v}^{\gamma} \otimes V_{v}^{2}\right)\right] \\
& \cdot \sum_{i<j}\left[u_{v} d x_{v}^{i} \wedge u_{v} d x_{v}^{j} \otimes \tau_{0}^{v} L\left(u_{v} e_{i}, u_{v} e_{j}\right)\right] d v
\end{aligned}
$$

Now if we consider $L_{x_{s}}$ as a $\xi_{x_{s}} \otimes \xi_{x_{s}}^{*}$ valued 2 form on $T_{x_{s}} M$, we have clearly

$$
L_{x_{s}}=\sum_{i<j} L\left(u_{v} e_{i}, u_{v} e_{j}\right) u_{v} d x^{i} \wedge u_{v} d x^{j}
$$

Let then $H_{s}$ be the solution of the differential equation

$$
d H_{s}=H_{s} \tau_{0}^{s} L_{x_{s}} d s, \quad H(0)=I .
$$

In (2.40) $H_{s}$ should be understood as a formal power series of even forms on $T_{x} \bar{M}^{0}$ valued in $\xi_{x_{0}} \otimes \xi_{x_{0}}^{*}$ in the sense that

$$
H_{s}=I+\int_{0}^{s} \tau_{0}^{v} L_{x_{v}} d v+\int_{0 \leqq v^{1} \leqq v^{2} \leqq s} \tau_{0}^{v^{1}} L_{x_{v^{1}}} \wedge \tau_{0}^{v^{2}} L_{x_{v^{2}}} d v^{1} d v^{2}+\ldots
$$


so that for instance if $X, Y, Z, T \in T_{x} \bar{M}^{\infty}$

$$
\begin{aligned}
& \left(\int_{0 \leqq v^{1} \leqq v^{2} \leqq s} \tau_{0}^{v^{1}} L_{x_{v^{1}}} \wedge \tau_{0}^{v^{2}} L_{x_{v^{2}}} d v^{1} d v^{2}\right)(X, Y, Z, T) \\
& =\int_{0 \leqq v^{1} \leqq v^{2} \leqq s}\left[\tau_{0}^{v^{1}} L_{x_{v 1}}\left(X_{v^{1}}, Y_{v^{1}}\right) \tau_{0}^{v^{2}} L_{x_{v 2}}\left(Z_{v^{2}}, T_{v^{2}}\right)\right. \\
& \left.+\tau_{0}^{v^{1}} L_{x_{v^{1}}}\left(Y_{v^{1}}, Z_{v^{1}}\right) \tau_{0}^{v^{2}} L_{x_{v^{2}}}\left(X_{v^{2}}, T_{v^{2}}\right)+\ldots\right] d v^{1} d v^{2} .
\end{aligned}
$$

It is then clear that (2.33) is exactly the formal power series of complex valued even forms $\operatorname{Tr} H_{1} \tau_{0}^{1}$. Using (2.29), (2.31), we get

$$
\operatorname{Ind} D_{+}=\exp \frac{n(n-1) t^{2}}{16} \frac{\left(\prod_{1}^{+\infty} m^{2}\right)^{l}}{(2 \pi)^{l}} i^{l} \int \exp \left(-\frac{E(x)+\sigma}{t}\right) \wedge \operatorname{Tr} H_{1} \tau_{0}^{1} \text {. }
$$

We now eliminate $\exp \frac{n(n-1) t^{2}}{16}$ by nonrigorous arguments.

In fact, assume that $\xi$ has complex dimension $1 . L_{x}$ is now a complex valued 2-form on $T_{x} M$. Consider the differential equation

$$
d U_{s}^{\prime}=-\frac{t}{2} U_{s}^{\prime}\left[\sum_{i<j}\left(u_{0} e_{i}\right)\left(u_{0} e_{j}\right) L_{x_{s}}\left(u_{s} e_{i}, u_{s} e_{j}\right)\right] d s ; \quad U_{0}^{\prime}=I
$$

In (2.44), $U_{s}^{\prime}$ acts on $F_{ \pm x_{0}}$. Clearly,

$$
\varrho_{+}\left(U_{1} \tau_{0}^{1}\right)-\varrho_{-}\left(U_{1} \tau_{0}^{1}\right)=\left[\chi_{+}\left(U_{1}^{\prime} \tau_{0}^{1}\right)-\chi_{-}\left(U_{1}^{\prime} \tau_{0}^{1}\right)\right]\left[\tau_{0}^{1}\right]_{\xi},
$$

where $\left[\tau_{0}^{1}\right]_{\xi}$ is the complex number which gives the parallel transport along $x$ on the considered fiber of $\xi$.

Let $\tau$ be the 2 -form on $T_{x} \bar{M}^{\infty}$ defined by

$$
\tau(Y, Z)=\int_{0}^{1} L_{x_{s}}\left(Y_{s}, Z_{s}\right) d s
$$

By using the same normalization prescriptions as in (2.15), we find

$$
\chi_{+}\left(U_{1}^{\prime} \tau_{0}^{1}\right)-\chi_{-}\left(U_{1}^{\prime} \tau_{0}^{1}\right)=\frac{ \pm i^{l}}{\left(\prod_{1}^{+\infty} 4 \pi^{2} m^{2}\right)^{l}} \operatorname{Pf}[-(\sigma+t \tau)] .
$$

We can then proceed as before and obtain formula (2.43), with the exception that $\exp \frac{n(n-1) t^{2}}{16}$ has disappeared.

This inconsistency comes from the fact that $\gamma$ is not differentiable.

The normalization prescriptions become ambiguous, and it is not unnatural that inconsistencies arise. It should be pointed out that $x$. itself being nondifferentiable, other severe inconsistencies may develop in the process. It is also plausible that the term $\exp \left(-t \int_{0}^{1} \frac{K\left(x_{s}\right) d s}{8}\right)$, which is omitted in (2.19), (2.43) does appear because of the nondifferentiability of $x$. 
In any case, since when $\xi$ is one dimensional, there is no need to introduce $\gamma$, and $\exp \frac{n(n-1) t^{2}}{16}$ does not appear in the final formula, we feel entitled to cancel it in (2.43) and rewrite (2.43) in the form

$$
\text { Ind } D_{+}=\frac{\left(\prod_{1}^{+\infty} m^{2}\right)^{l} i^{l}}{(2 \pi)^{l}} \int \exp \left(-\frac{E(x)+\sigma}{t}\right) \wedge \operatorname{Tr} H_{1} \tau_{0}^{1} .
$$

Remark 2. Formula (2.48) is, of course, formal. However, as we shall see in the next section, the right-hand side is still the integral of an equivariantly closed form.

\section{Equivariant Cohomology on the Loop Space and the Chern Character}

In this section, we prove that the integrand in the formal index formula which we derived in Sect. 2 is indeed equivariantly closed. It can be obtained as an equivariant characteristic class over an infinite dimensional bundle over the loop space. It is calculated by integrating a differential equation over the trajectory of each considered loop.

In a) the bundle $\bar{X}^{\infty}$ of periodic sections of $X$ over each loop is introduced, whose structure group is the loop space of $U(k)$.

In b) an equivariant cohomology class is constructed on the loop space by following the general prescriptions of Chern-Weil theory $[4,10]$ which exactly coincides with what has been found in Sect. 2. The restriction of this class to the base manifold $M$ is exactly the Chern character of $\xi$. In c) the obvious formal application is done to the index theorem. In d), we show briefly how to include the Lefschetz fixed point formulas of Atiyah and Bott [2] and Atiyah and Singer [3] in this framework.

a) Assumptions and Notations. The assumptions and notations are essentially the same as in Sects.1 and 2. In particular, we will use the results of Sect. 2a).

Definition 3.1. $\overline{U(k)}^{\infty}$ [respectively $\overline{\mathscr{U}(k)^{\infty}}, \bar{X}^{\infty}$ ] denotes the set of $C^{\infty}$ mappings from $S_{1}=R / Z$ into $U(k)$ [respectively $\left.\mathscr{U}(k), X\right]$. We endow $\bar{M}^{\infty}, \bar{U}(k)^{\infty}, \overline{\mathscr{U}}^{\infty}(k), \bar{X}^{\infty}$ with the topology of uniform convergence of the considered functions and their derivatives over $S_{1}$.

$\overline{U(k)}^{\infty}$ is naturally a group, with the obvious multiplication rule $(a b)_{s}=a_{s} b_{s}$, $\overline{\mathscr{U}(k)})^{\infty}$ is, at least formally, the Lie algebra of $\overline{U(k)}^{\infty}$.

Let $\pi^{\prime}$ be the projection $X \rightarrow M$. $\pi^{\prime}$ obviously extends to a projection $\bar{X}^{\infty} \rightarrow \bar{M}^{\infty}$.

Moreover, if $a \in \overline{U(k)}^{\infty}, r \in \bar{X}^{\infty}$, we may define $r a$ in the obvious way.

We now have

Theorem 3.2. $\bar{X}^{\infty}$ is a $\overline{U(k)}^{\infty}$ principal bundle over $\bar{M}^{\infty}$.

Proof. Take $x \in \bar{M}^{\infty}$. We first exhibit $r \in \bar{X}^{\infty}$ such that $\pi^{\prime} r=x$. Take $v_{0} \in X_{x_{0}}$, and let $v_{s}$ be the parallel transport of $v_{0}$ along $x$. If $r \in \bar{X}^{\infty}$, we may write $r_{s}=v_{s} a_{s}$ with $a_{s} \in U(k)$ for each $s \in R / Z$.

Set $b=v_{1}^{-1} v_{0}$. Of course, $b \in U(k)$. Since $U(k)$ is connected, we can find $a_{s}$ with values in $U(k)$ which is such that 
a) It is $C^{\infty}$ on $] 0,1\left[\right.$, constant on $\left[0, \frac{1}{4}\right]$ and $\left[\frac{3}{4}, 1\right]$.

b) $a_{s}$ is equal to $e$ on $\left[0, \frac{1}{4}\right]$ and to $b$ on $\left[\frac{3}{4}, 1\right]$.

We have then found one $r \in \bar{X}^{\infty}$ such that $\pi^{\prime} r=x$. Clearly, the other elements of $\bar{X}_{x}^{\infty}$ are obtained by the action of $\overline{U(k)^{\infty}}$ on $r$.

To prove the bundle property, we only need to prove local triviality. Using the exponential mapping in $M$ and parallel transport in $X$, local triviality is very easy to prove. This is left to the reader.

If $r \in \bar{X}^{\infty}$, the tangent space $T_{r} \bar{X}^{\infty}$ is the space of smooth periodic vector fields $Y$ over $r$ so that $Y_{s} \in T_{r_{s}} X$.

Definition 3.3. $\bar{\lambda}$ denotes the $\overline{\mathscr{U}(k)^{\infty}}$ valued connection form on $\bar{X}^{\infty}$ such that if $r \in \bar{X}^{\infty}, Y \in T_{r} \bar{X}^{\infty}$,

$$
\bar{\lambda}(Y)_{s}=\lambda\left(Y_{s}\right)
$$

If $\bar{\Lambda}$ is the curvature form of the considered connection, if $r \in \bar{X}^{\infty}, Y, Z \in T_{r} \bar{X}^{\infty}$, then

$$
\bar{\Lambda}(Y, Z)_{s}=\Lambda\left(Y_{s}, Z_{s}\right) .
$$

b) Equivariant Characteristic Classes over $\bar{M}^{\infty}$ and the Chern Character. Observe that for $s \in S_{1}$, we can define the action of $k_{s}, k_{s}^{\prime}$ on $\bar{X}^{\infty}$ and $\left.\overline{U(k)}\right)^{\infty}$ by

$$
k_{s}(r)=r_{s+.}, \quad k_{s}^{\prime}(a)=a_{s+\cdot}
$$

$k_{s}$ is a group of transformations of $\bar{X}^{\infty}$ generated by the vector field $K$ given by

$$
K(r)=\frac{d r}{d s} .
$$

$k_{s}^{\prime}$ is a group of isomorphisms of $\overline{U(k)^{\infty}}$. Moreover, $k_{s}(r a)=k_{s}(r) k_{s}^{\prime}(a) . k_{s}$ is then a bundle isomorphism of $\bar{X}^{\infty}$. However, it does not act trivially on the structure group $\overline{U(k)}{ }^{\infty}$, so that some care will have to be taken when using the results of [10].

In particular, the connection form $\bar{\lambda}$ is not invariant under $k_{s}$. To be more precise, we now define

Definition 3.4. $\lambda^{\prime}$ is the $\overline{\mathscr{U}(k)^{\infty}}$ valued form defined by the fact that if $Y \in T_{r} \bar{X}^{\infty}$

$$
\left[\bar{\lambda}^{\prime}(Y)\right]_{s}=\frac{d}{d s} \bar{\lambda}(Y)
$$

$J_{K}$ is the $\overline{\mathscr{U}(k)^{\infty}}$ valued function defined by

$$
J_{K}=\bar{\lambda}(K) \text {. }
$$

If $\alpha$ is a differential form, $D \alpha$ denotes the form which, when acting on horizontal vectors, coincides with $d \alpha$, and which is 0 if any of the vectors on which it acts is vertical. Also if $a \in \overline{U(k)})^{\infty}, R_{a}$ denotes the usual action of the mapping $r \rightarrow r a$ on the tensor algebra.

We now have the following relations, which slightly differ from [10]. 
Theorem 3.5. The following relations hold

$$
\begin{aligned}
& L_{K} \bar{\lambda}=\bar{\lambda}^{\prime}, \\
& D J_{K}=d J_{K}+\left[\bar{\lambda}, J_{K}\right]-\bar{\lambda}^{\prime}, \\
& D J_{K}+i_{K} \bar{\Lambda}=0 .
\end{aligned}
$$

Moreover, if $a \in \overline{U(k)}^{\infty}$, then

$$
\left(R_{a} J_{K}\right)_{s}=\operatorname{ad}\left(a_{s}^{-1}\right) J_{K}+a_{s}^{-1} \dot{a}_{s} .
$$

Proof. Since

$$
\left(R_{a} J_{K}\right)(r)=J_{K}(r a),
$$

we have

$$
R_{a} J_{K}=\bar{\lambda}\left[\frac{d(r a)}{d t}\right]
$$

and so (3.6) holds.

Moreover, if $Y \in T_{r} \bar{X}^{\infty}$, we have

$$
\bar{\lambda}\left(k_{s^{*}} Y\right)=k_{s}^{\prime} \bar{\lambda}(Y),
$$

so that

$$
\left(L_{K} \bar{\lambda}\right)(Y)=\bar{\lambda}^{\prime}(Y)
$$

Take $A \in \overline{\mathscr{U}(k)})^{\infty}$ and let $A^{*}$ be the vertical vector field such that $\bar{\lambda}\left(A^{*}\right)=A$. Clearly,

$$
K\left[\bar{\lambda}\left(A^{*}\right)\right]=0,
$$

so that using (3.10), we get

$$
\bar{\lambda}\left(\left[K, A^{*}\right]\right)=-\bar{\lambda}^{\prime}\left(A^{*}\right)
$$

Now

$$
A^{*}\left[J_{K}\right]=\left(L_{A^{*}} \bar{\lambda}\right)(K)+\bar{\lambda}\left(\left[A^{*}, K\right]\right) .
$$

Using (1.3) we also know that

$$
\left(L_{A^{*}} \bar{\lambda}\right)(K)=\left[J_{K}, A\right] .
$$

From (3.12)-(3.14) we see that the second line in (3.5) holds.

Finally, we know that

$$
L_{K} \bar{\lambda}=d i_{K} \bar{\lambda}+i_{K} d \bar{\lambda}=d J_{K}-\left[J_{K}, \bar{\lambda}\right]+i_{K} \bar{\Lambda} .
$$

Using the first two lines of (3.5) and (3.15), we see that the third line in (3.5) holds.

Remark 1. The third line in (3.5) is also an easy consequence of the very definition of the curvature tensor $\Lambda$.

We now set a definition which closely imitates (2.40). 
Definition 3.6. $H_{s}^{\prime}$ denotes the solution of the differential equation

$$
H_{s}^{\prime}=I+\int_{0}^{s} H_{v}^{\prime}\left(J_{K}(v)+\bar{\Lambda}_{x_{v}}\right) d v .
$$

Of course, $\bar{\Lambda}$ is here considered as a 2 -form and $H_{s}^{\prime}$ should be considered as a formal power series of matrix valued even forms.

We now have

Theorem 3.7. For any $s \in[0,1]$,

$$
\left[D+i_{K}\right] H_{s}^{\prime}=0 .
$$

Moreover, if $a \in \overline{U(k)}^{\infty}$,

$$
R_{a}\left(H^{\prime}\right)_{s}=a_{0}^{-1} H_{s}^{\prime} a_{s}
$$

Proof. Since

$$
H_{s}^{\prime}=I+\int_{0}^{s} H_{v}^{\prime}\left(J_{K}+\bar{\Lambda}\right) d v
$$

we have

$$
\left(D+i_{K}\right) H_{s}^{\prime}=\int_{0}^{s}\left[\left(D+i_{K}\right) H_{v}^{\prime}\right]\left(J_{K}+\bar{\Lambda}\right) d v+\int_{0}^{s} H_{v}^{\prime}\left(D+i_{K}\right)\left(J_{K}+\bar{\Lambda}\right) d v .
$$

Using the third line in (3.5), (3.17) follows. Moreover, from (3.6), we know that

$$
R_{a}\left(J_{K}+\bar{\Lambda}\right)=\operatorname{ad}\left(a_{s}^{-1}\right)(J+\bar{\Lambda})+a_{s}^{-1} \dot{a}_{s} .
$$

Equation (3.18) is now obvious.

In particular, in (3.18), since $a_{1}=a_{0}$, we see that $\left(R_{a} H^{\prime}\right)_{1}=a_{0}^{-1} H_{1}^{\prime} a_{0}$, so that $\operatorname{Tr} H_{1}^{\prime}$ is invariant under the action of $\left.\overline{U(k)}\right)^{\infty}$. $\operatorname{Tr} H_{1}^{\prime}$ is clearly a horizontal form. We can then define

Definition 3.8. $\beta$ denotes the formal series of even forms on $\bar{M}^{\infty}$ such that

$$
\operatorname{Tr} H_{1}^{\prime}=\pi^{*} \beta .
$$

We now have the fundamental result.

Theorem 3.9. $\beta$ is equivariantly closed. Moreover, the equivariant cohomology class of $\beta$ does not depend on the connection on $X$. The restriction of $\beta$ to $M$ is equal to $\operatorname{Tr} \exp \Lambda$.

Proof. By Theorem 3.7, we know that $\left(D+i_{K}\right) \pi^{*} \beta=0$. Since $\left(D+i_{K}\right) \pi^{*} \beta$ $=\pi^{*}\left[\left(d+i_{X}\right) \beta\right]$, it is clear that $\left(d+i_{X}\right) \beta=0$. To prove the second part of the theorem, we proceed very much as in [10]. Let $\lambda^{\prime}$ be another connection form on $X$. Set

$$
\alpha=\lambda^{\prime}-\lambda, \quad \lambda^{t}=\lambda+t \alpha .
$$

$\alpha$ is a tensorial 1-form, so that if $D^{t}$ denotes the operator $D$ corresponding to $\lambda^{t}$,

$$
D^{t} \alpha=d \alpha+\alpha \wedge \lambda^{t}+\lambda^{t} \wedge \alpha .
$$


$\bar{\alpha}, \bar{\lambda}^{t}, \bar{\Lambda}^{t}, J_{K}^{t}$ are defined in the obvious way. Let $H^{t}$ be the solution of the equation

$$
H_{s}^{t}=I+\int_{0}^{s} H_{v}^{t}\left(J_{K}^{t}+\bar{\Lambda}^{t}\right) d v
$$

Clearly,

$$
\frac{\partial J_{K}^{t}}{\partial t}=i_{K} \alpha ; \quad \frac{\partial \Lambda^{t}}{\partial t}=d \alpha+\alpha \wedge \lambda^{t}+\lambda^{t} \wedge \alpha,
$$

so that using (3.24), we get

$$
\frac{\partial H_{s}^{t}}{\partial t}=\int_{0}^{s} \frac{\partial H_{v}^{t}}{\partial t}\left[J_{K}^{t}+\bar{\Lambda}^{t}\right] d v+\int_{0}^{s} H_{v}^{t t}\left(D^{t}+i_{K}\right) \bar{\alpha} d v .
$$

Let $Z_{s}^{t}$ be the solution of

$$
Z_{s}^{t}=I+\int_{0}^{s}\left[Z_{v}^{t}\left(J_{K}^{t}+\bar{\Lambda}^{t}\right)+H_{v}^{t t} \bar{\alpha}\right] d v
$$

Set

$$
Z_{s}^{t}=\left(D^{t}+i_{K}\right) Z_{s}^{t}
$$

Using Theorems 3.5 and 3.7, we know that

$$
Z_{s}^{t}=\int_{0}^{s}\left[Z_{v}^{t}\left(J_{K}^{t}+\bar{\Lambda}^{t}\right)+H_{v}^{t}\left(D^{t}+i_{K}\right) \bar{\alpha}\right] d v .
$$

Comparing (3.27) and (3.30) we see that

$$
\frac{\partial H_{s}^{t}}{\partial t}=\left(D^{t}+i_{K}\right) Z_{s}^{t}
$$

Moreover, using (3.28), and Theorems 3.5 and 3.7, we see that

$$
R_{a} Z_{s}^{t}=a_{0}^{-1} Z_{s}^{t} a_{s}
$$

The form $\operatorname{Tr} Z_{1}^{t}$ is then $\bar{U}(k)^{\infty}$ invariant, horizontal, and so projects as a form $\gamma^{t}$ on $\bar{M}^{\infty}$. Using (3.31), we see that

$$
\frac{\partial \beta^{t}}{\partial t}=\left(d+i_{X}\right) \gamma^{t}
$$

and so

$$
\beta^{\prime}-\beta=\left(d+i_{X}\right) \int_{0}^{1} \gamma^{t} d t
$$

To compute the restriction of $\beta$ to $M$, note that on $M, J_{K}=0$, and that $\bar{\Lambda}$ is constant on each trivial path $x_{0}$, so that $H_{1}^{\prime}=\exp \Lambda$. The theorem follows.

Remark 2. By slightly modifying the construction of $\beta$, we may obtain $\beta$ as a "standard" equivariant characteristic class. In fact, let $\overline{V(k)})^{\infty}$ be the semi-direct product of $\overline{U(k)^{\infty}}$ and $S_{1}$, with the multiplication rule, $(a, s)\left(a^{\prime}, s^{\prime}\right)=\left(a k_{s}^{\prime}\left(a^{\prime}\right), s+s^{\prime}\right)$. The Lie algebra $\overline{\mathscr{V}(k)})^{\infty}$ of $\overline{V(k)^{\infty}}$ is an affine Lie algebra associated with $\mathscr{U}(k)$ in the 
sense of Kac [13] and Frenkel [12]. It identifies naturally with the Lie algebra of differential operators on $S_{1}$,

$$
b \frac{d}{d t}+A, \quad b \in R, \quad A \in \overline{\mathscr{U}(k)^{\infty}} .
$$

Set $\bar{Z}^{\infty}=\bar{X}^{\infty} \times S_{1}$. Then $\bar{Z}^{\infty}$ is naturally a $\overline{V(k)}{ }^{\infty}$ bundle over $\bar{M}^{\infty}$ with the product

$$
(r, s)\left(a, s^{\prime}\right)=\left(r k_{s}^{\prime}(a), s+s^{\prime}\right) \text {. }
$$

$\bar{X}^{\infty}$ embeds naturally in $\bar{Z}^{\infty}$ by $r \in \bar{X}^{\infty} \rightarrow(r, 0) \in \bar{Z}^{\infty}$, which is a bundle homomorphism. The connection form $\bar{\lambda}$ on $\bar{X}^{\infty}$ can then be naturally extended to a connection form $\bar{\mu}$ on $\bar{Z}^{\infty}$. Namely, if $(Y, b) \in T_{(r, s)} \bar{Z}^{\infty}$, using the identification (3.35), we have

$$
\tilde{\mu}(Y, b)=b \frac{d}{d t}+k_{-s}^{\prime}[\bar{\lambda}(Y)] .
$$

If $\bar{N}$ is the curvature form of $\bar{\mu}$ we deduce from (3.37) that at $(r, s)$,

$$
\bar{N}=k_{-s}^{\prime}(\bar{\Lambda}) \text {. }
$$

$S_{1}$ acts naturally on $\bar{Z}^{\infty}$ by the formula

$$
k_{s}^{\prime \prime}(r, t)=\left(k_{s} r, s+t\right) .
$$

Using (3.36), (3.38), for any $s \in S_{1}=R / Z, k_{s}^{\prime \prime}$ is a bundle isomorphism, i.e.

$$
k_{s}^{\prime \prime}\left[(r, t)\left(a, s^{\prime}\right)\right]=\left[k_{s}^{\prime \prime}(r, t)\right]\left(a, s^{\prime}\right),
$$

and $k_{s}^{\prime \prime}$ preserves the connection $\bar{\mu}$. $k_{s}^{\prime \prime}$ is a group of bundle isomorphisms of $\bar{Z}^{\infty}$. It is generated by the vector field $K^{\prime \prime}$ given by

Set

$$
K^{\prime \prime}=(K, 1) \text {. }
$$

Obviously, at $(r, s)$,

$$
J_{K^{\prime \prime}}=\bar{\mu}\left(K^{\prime \prime}\right) .
$$

$$
J_{K^{\prime \prime}}=\frac{d}{d t}+k_{-s}^{\prime}[\bar{\lambda}(K)]
$$

It then follows that at $(r, s)$,

$$
J_{K^{\prime \prime}}+\bar{N}=\frac{d}{d t}+k_{-s}^{\prime}[\bar{\lambda}(K)+\bar{\Lambda}] .
$$

Of course, $J_{K^{\prime \prime}}+\bar{N}$ must be considered as a differential form on $\bar{Z}^{\infty}$ with values in $\overline{\mathscr{V}(k)})^{\infty}$.

Consider now the differential equation on $[0,1]$,

$$
\left(J_{K^{\prime \prime}}+\bar{N}\right)\left(H^{\prime \prime}\right)=0 ; \quad H^{\prime \prime}(0)=I,
$$

which can also be written as

$$
\frac{d H^{\prime \prime}}{d t}+\left\{k_{-s}^{\prime}[\bar{\lambda}(K)+\bar{\Lambda}]\right\} H^{\prime \prime}=0 ; \quad H^{\prime \prime}(0)=I
$$


Clearly, in the sense of formal power series, at $(r, 0)$,

so that at $(r, 0)$,

$$
H^{\prime}=\left[H^{\prime \prime}\right]^{-1},
$$

$$
\operatorname{Tr}\left[H_{1}^{\prime \prime}\right]^{-1}=\pi^{*} \beta .
$$

At least formally, this permits us to construct $\beta$ as a standard equivariant characteristic class by extending Chern-Weil's theory as in Berline and Vergne [10]. However, because the problem is now infinite dimensional, we cannot directly use the argument in [10] to deduce "trivially" the main properties of $\beta$. So even using affine Lie algebras, the results in Theorems 3.5 and 3.7 would still have to be proved in another form. Finally, note that the ad $\overline{V(k)})^{\infty}$ invariance of $\operatorname{Tr}\left[H_{1}^{\prime \prime}\right]^{-1}$ is very easy to prove.

Remark 3. The restriction of $\boldsymbol{\beta}$ to $M$ coincides, up to irrelevant normalization constants, with the Chern character of $\xi$.

Before going back to the index problem, let us make a final remark.

Namely, assume that $S_{1}$ acts on $M$ as a $C^{\infty}$ group of diffeomorphisms, and that this action lifts to $\xi$. There is then a natural mapping $f: M \rightarrow \bar{M}^{\infty}$, which to each $x$ associates the orbit of $x$ by $S_{1}$.

Let $Y$ be the vector field on $M$ which generates the action of $S_{1}, Z$ the corresponding vector field in $\bar{X}^{\infty}$, so that $\pi_{*}^{\prime} Z=Y$.

Theorem 3.10. $f^{*} \beta$ is equivariantly closed, i.e. $\left(d+i_{Y}\right) f^{*} \beta=0$. If $\lambda^{\prime}$ is a $S_{1}$ invariant connection on $\xi$, and if $\Lambda^{\prime}$ is the curvature, the equivariant cohomology class of $f^{*} \beta$ is equal to $\operatorname{Tr} \exp \left[\lambda^{\prime}(Z)+\Lambda^{\prime}\right]$.

Proof. Since $f_{*} Y=X$, and since $\left(d+i_{X}\right) \beta=0$, the first part of the theorem is clear. The equivariant cohomology class of $\beta$ does not depend on $\lambda$. Since $S_{1}$ is compact, there is always an $S_{1}$ invariant connection on $\xi$. Then $\lambda^{\prime}(Z)$ is constant on an orbit $f(x)$. A similar argument for $\Lambda^{\prime}$ shows that $f^{*} \beta=\operatorname{Tr} \exp \left[\lambda^{\prime}(Z)+\Lambda^{\prime}\right]$.

c) The Index Theorem. Note that if $H^{\prime}$ is calculated at $r \in \bar{X}^{\infty}$, then $H_{s}^{\prime}=r_{0}^{-1} H_{s} \tau_{0}^{s} r_{s}$. It is now clear that formula (2.48) becomes

$$
\operatorname{Ind} D_{+}=\frac{\left(\prod_{1}^{+\infty} m^{2}\right)^{l}}{(2 \pi)^{l}} i^{l} \int \exp \left(-\frac{E(x)+\sigma}{t}\right) \wedge \beta .
$$

The form under the integral is now globally equivariantly closed.

Let $e$ be the equivariant Euler class of the normal bundle of $M$. This is an infinite product which diverges (see [1]).

If we apply formally the classical formulas of equivariant cohomology, we get

$$
\operatorname{Ind} D_{+}=\int_{M} \frac{\left(\Pi m^{2}\right)^{l}}{(2 \pi)^{l}} i^{1} \frac{1}{e} \wedge \operatorname{Tr} \exp \Lambda .
$$

By proceeding as in Atiyah [1] for the first term, we get

$$
\operatorname{Ind} D_{+}=\int_{M} \prod_{1}^{l} \frac{x_{i} / 2}{\sinh x_{i} / 2}(T M) \wedge \operatorname{ch} \xi,
$$

which is the correct formula. 
Everything which we have done can be made to work rigorously in at least one case.

Namely, consider the vector bundle $L_{\infty}$ over $M$ whose fiber at $x_{0}$ is the set of $C^{\infty}$ mappings $s \in S_{1}=R / Z \rightarrow y_{s} \in T_{x_{0}}^{M}$. Let $\pi$ be the projection $L_{\infty} \rightarrow M$.

Using the Levi-Civita connection, we may decompose $T(T M)$ into an horizontal and a vertical part.

If $Y \in T_{y} L_{\infty}$, we denote by $Y_{s}^{v}$ the vertical part of $Y_{s}$. Let $\eta$ be the 1-form on $L_{\infty}$ defined by

$$
\eta(Y)=\int_{0}^{1}\left\langle\dot{y}_{s}, Y_{s}^{v}\right\rangle d s
$$

Then it is trivial to check that

$$
\frac{d \eta}{2}(Y, Z)=\int_{0}^{1}\left\langle\frac{d Y^{v}}{d s}, Z_{s}^{v}\right\rangle d s+\int_{0}^{1} \frac{1}{2}\left\langle R\left(\pi^{*} Y, \pi^{*} Z\right) y_{s}, \dot{y}_{s}\right\rangle d s .
$$

Let $X$ be the vertical vector field such that

$$
X(y)_{s}=\frac{d y}{d s} .
$$

Set

$$
E(y)=\int_{0}^{1} \frac{|\dot{y}|^{2}}{2} d s, \quad \sigma=\eta / 2 .
$$

Clearly,

$$
L_{x} \eta=0
$$

and moreover,

$$
\left(d+i_{X}\right) \frac{\eta}{2}=E+\sigma .
$$

For $x_{0} \in M$, let $P_{1}^{x_{0}}$ be the law of the Brownian bridge $w^{1}$ in $T_{x_{0}} M$, with $w_{0}^{1}=w_{1}^{1}=0$. In Theorems 3.15 and 3.18 in [7] we proved that

$$
\operatorname{Ind} D_{+}=\int_{M} \int_{\exp }\left\{\left\{-\frac{i}{4 \pi} \int_{0}^{1} R\left(d w_{s}^{1}, w_{s}^{1}\right)\right\} d P_{1}^{x} \wedge \operatorname{ch} \xi,\right.
$$

where $-\frac{i}{4 \pi} \int_{0}^{1} R\left(d w_{s}^{1}, w_{s}^{1}\right)$ is considered as an antisymmetric matrix whose exponential is taken in the exterior algebra of $M$. Using the symmetries of $R$ as in [7, Proposition 3.16], we may put (3.57) in the form

$$
\operatorname{Ind} D_{+}=\frac{\left(\prod_{1}^{+\infty} m^{2}\right)^{l}}{(2 \pi)^{l}} i^{l} \int_{L_{\infty}} \exp -\frac{E(y)+\sigma}{t} \wedge \operatorname{Tr} \exp \Lambda,
$$

where the renormalizations in (3.58) are essentially trivial. However, for obvious reasons, it is totally unnecessary to use equivariant cohomology to calculate (3.58)! 
d) Extensions. Let $Y$ be a Killing vector field on $M$. Assume that $Y$ lifts to a $U(k)$ invariant vector field $Z$ on $X$, which preserves the connection form $\lambda$.

We now consider the problem of calculating the Lefschetz number $L\left(e^{Z}\right)$. We show briefly how to put this problem in the form suggested by Witten and Atiyah [1]. We use the notations of Sect. 2.

Let $\alpha^{\prime}$ be the 1 -form on $\bar{M}^{\infty}$ defined by

$$
A \in T_{x} \bar{M}_{\infty} \rightarrow \alpha^{\prime}(A)=\int_{0}^{1}\left\langle X_{s}+Y\left(x_{s}\right), A_{s}\right\rangle d s
$$

(recall that $\left.X(x)=\frac{d x}{d s}\right)$. Since $Y$ is a Killing vector field, it is easy to check that

$\frac{d \alpha^{\prime}}{2}$ is the two form $\sigma^{\prime}$ defined by $L_{X+Y} \alpha^{\prime}=0$.

$$
\sigma^{\prime}(A, B)=\int_{0}^{1}\left(\left\langle\frac{D A}{D s}+\nabla_{A} Y, B\right\rangle\right) d s
$$

$\frac{i_{X+Y} \alpha^{\prime}}{2}$ is the functional $E^{\prime}$ defined by

$$
E^{\prime}(x)=\int_{0}^{1} \frac{|\dot{x}+Y|^{2}}{2} d s
$$

Equivariant cohomology will then be defined with respect to the vector field $X+Y$.

Similarly, the definition of $J_{K}$ in Definition 3.4 is changed into

$$
J_{K+Z}(s)=\bar{\lambda}(K+Z)
$$

$H^{\prime}$ is now calculated with $J_{K+Z}$ instead of $J_{K}$, and $\beta$ is changed into $\beta^{\prime}$.

Using the results in [7] on the probabilistic representation of the Lefschetz number, the Girsanov transformation [6], and proceeding as previously, it is not difficult to obtain the formal formula

$$
L\left(e^{Z}\right)=\frac{\left(\prod_{1}^{+\infty} m^{2}\right)^{l}}{(2 \pi)^{l}} i^{l} \int \exp -\left(\frac{E^{\prime}(x)+\sigma^{\prime}}{t}\right) \wedge \beta^{\prime}
$$

(we use the sign conventions of [7] for the definition of $L\left(e^{Z}\right)$ ).

Now the zeroes of $X+Y$ are exactly the closed orbits of $Y$, which correspond in an obvious way to the fixed points of $e^{Y}$.

In the case, where the fixed points of $e^{Y}$ coincide with the zeroes of $Y$, the formal application of the fixed point formula in equivariant cohomology gives immediately the Lefschetz fixed point formulas of Atiyah and Bott [2] and Atiyah and Singer [3].

If $e^{Y}$ has other fixed points than the zeroes of $Y$, this is also the case. To see this, one must note that $\Omega$ is invariant under $Z$, so that the equivariant Euler class of the normal bundle of the fixed loops can be calculated "pointwise" on the fixed point set of $e^{Y}$ in $M$. Similarly, the analogue of Theorem 3.10 shows that the "orbital" equivariant Chern character coincides with the usual equivariant Chern character.

Acknowledgements. I am very grateful to the referee for his comments and suggestions. Also I am indebted to $\mathrm{M}$. Vergne for helpful remarks. 


\section{References}

1. Atiyah, M.F.: Circular symmetry and stationary phase approximation. In: Proceedings of the conference in honor of L. Schwartz. Paris: Astérisque 1984

2. Atiyah, M.F., Bott, R.: A Lefschetz fixed point formula for elliptic complexes. I. Ann. Math.86, 374-407 (1967); II. 88, 451-491 (1968)

3. Atiyah, M.F., Singer, I.M.: The index of elliptic operators. I. Ann. Math. 87, 484-530 (1968); III. Ann. Math. 87, 546-604 (1968)

4. Atiyah, M.F., Bott, R.: The moment map and equivariant cohomology. Topology 23, 1-28 (1984)

5. Atiyah, M.F.: Elliptic operators, discrete groups, and Von Neumann algebras. Astérisque 32/33, 43-72 (1976)

6. Bismut, J.-M.: Transformations différentiables du mouvement Brownien. In: Proceedings of the conference in honor of L. Schwartz. Paris: Astérisque 1984

7. Bismut, J.-M.: The Atiyah-Singer theorems. A probabilistic approach. I. The index theorem. J. Funct. Anal. 57, 56-99 (1984); II. The Lefschetz fixed point formulas. J. Funct. Anal. 57, 329-348 (1984)

8. Bismut, J.-M.: Large deviations and the Malliavin calculus. In: Progress in Mathematics, Vol. 45. Boston: Birkhäuser 1984

9. Bismut, J.-M.: Mécanique aléatoire. In: Lecture Notes in Mathematics, Vol. 866. Berlin, Heidelberg, New York: Springer 1981

10. Berline, N., Vergne, M.: Zéros d'un champ de vecteur et classes caractéristiques équivariantes. Duke Math. J. 50, 539-549 (1983)

11. Duistermaat, J.J., Heckman, G.: On the variation of the cohomology of the reduced phase space. Invent. Math. 69, 259-268 (1982); Addendum 72, 153-158 (1983)

12. Frenkel, I.B.: Orbital theory for affine Lie algebras. Invent. Math. (to appear, 1984)

13. Kac, V.G.: Infinite dimensional Lie algebras. In: Progress in Mathematics, Vol. 44. Boston: Birkhäuser 1984

14. Witten, E.: Supersymmetry and Morse theory. J. Diff. Geom. 17, 661-692 (1982)

Communicated by A. Jaffe

Received April, 19, 1984; in revised form October 1, 1984 
